Article

\title{
Synthesis of 1,2,3-Triazole Derivatives and in Vitro Antifungal Evaluation on Candida Strains
}

Reginaldo G. Lima-Neto ${ }^{1}$, Nery N. M. Cavalcante ${ }^{2}$, Rajendra M. Srivastava ${ }^{2}$, Francisco J. B. Mendonça Junior ${ }^{3}$, Almir G. Wanderley ${ }^{4}$, Rejane P. Neves ${ }^{1}$ and Janaína V. dos Anjos ${ }^{2, *}$

1 Laboratório de Micologia Médica, Centro de Ciências Biológicas, Universidade Federal de Pernambuco (UFPE), 50670-901, Recife, PE, Brazil

2 Laboratório de Síntese Orgânica, Departamento de Química Fundamental, Universidade Federal de Pernambuco (UFPE), 50740-560, Recife, PE, Brazil

3 Laboratório de Síntese e Vetorização de Substâncias Bioativas, Universidade Estadual da Paraíba (UEPB), 58058-420, João Pessoa, PB, Brazil

4 Laboratório de Farmacologia Pré-Clínica e Toxicologia de Produtos Bioativos, Departamento de Fisiologia e Farmacologia, Universidade Federal de Pernambuco (UFPE), 50670-901, Recife, PE, Brazil

* Author to whom correspondence should be addressed; E-Mail: janaina.anjos@ufpe.br; Tel.: +55-81-2126-7411; Fax: +55-81-2126-8440.

Received: 12 April 2012; in revised form: 3 May 2012 / Accepted: 5 May 2012 / Published: 16 May 2012

Abstract: 1,2,3-Triazoles have been extensively studied as compounds possessing important biological activities. In this work, we describe the synthesis of ten 2-(1-aryl-1H1,2,3-triazol-4-yl)propan-2-ols via copper catalyzed azide alkyne cycloaddition (CuAAc or click chemistry). Next the in vitro antifungal activity of these ten compounds was evaluated using the microdilution broth method against 42 isolates of four different Candida species. Among all tested compounds, the halogen substituted triazole 2-[1-(4-chlorophenyl)- $\mathrm{H}$ $(1,2,3)$ triazol-4-yl]propan-2-ol, revealed the best antifungal profile, showing that further modifications could be done in the structure to obtain a better drug candidate in the future.

Keywords: click chemistry; 1,2,3-triazoles; Candida spp.; antifungal activity 


\section{Introduction}

Deep and superficial fungal infections have increased significantly over the past few decades. Control of fungal disease has proved to be difficult because of several risk factors. The number of patients at highest risk for these infections has been steadily increasing, especially among patients immunocompromised due to AIDS, organ transplantation, chemotherapy or other invasive procedures [1]. Because of this, there is a clear need for the development of effective antimycotic therapeutic agents for the treatment of fungal infections, since the major classes of antifungal drugs available have encountered resistance in clinical use [2,3]. Among these classes, azoles are the most used because of their broad spectrum, high potency and low toxicity [4].

Azoles are competitive inhibitors of lanosterol $14 \alpha$-demethylase (a cytochrome P-450 enzyme), leading to a decrease in the fungal biosynthesis of ergosterol, which is a key compound of fungal cell membranes, thereby preventing fungal growth [5,6]. Beyond the antifungal properties [7-9], triazoles possess a variety of interesting biological activities, forming part of the scaffolds of antibacterial and antituberculosis agents [10-14], neuraminidase inhibitors [15], anticancer compounds [16], antiviral agents [17], analgesic compounds [18], herbicides [19] and plant growth regulators [20].

Considering the above mentioned advantages of triazole-containing antifungal drugs and the increasing drug resistance mechanisms in these type of microorganisms, we decided to synthesize 2-(1-aryl-1H-1,2,3-triazol-4-yl)propan-2-ols capable of inhibiting cell growth of some Candida species with clinical relevance and testing their activity using the microdilution broth method.

\section{Results and Discussion}

\subsection{Chemistry}

Prior to the synthesis of the 2-(1-aryl-1H-1,2,3-triazol-4-yl)propan-2-ols, the aromatic azides $\mathbf{2 a - j}$ were prepared from the corresponding anilines $1 \mathbf{1 a}-\mathbf{j}$ following the Sandmeyer conditions [21]. The aromatic azides were then reacted with 2-methylbut-3-yn-2-ol (3) using $\mathrm{Cu}(\mathrm{OAc})_{2}$ and sodium ascorbate as catalyst in 1:1 dichloromethane:water [22,23] to give the products $\mathbf{4 a - j}$ in good yields (Scheme 1).

Scheme 1. Synthesis of triazoles $\mathbf{4 a - j}$.
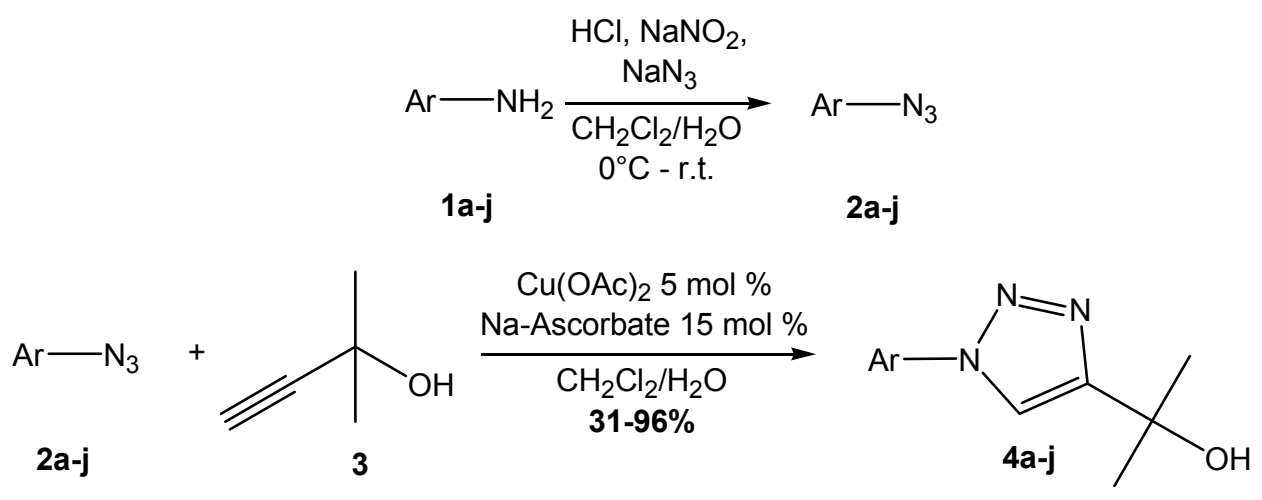
a: Phenyl
f: $m-\mathrm{NO}_{2}-\mathrm{Phenyl}$
b: $p$-Tolyl
g: $p-\mathrm{NO}_{2}-$ Phenyl
c: $p$-Cl-Phenyl
h: 3,4-di- $\mathrm{CH}_{3}$-Phenyl
d: $p$-Br-Phenyl
i: alpha-Naphthyl
e: $p-\mathrm{OCH}_{3}-$ Phenyl $\mathbf{j}$ : beta-Naphthyl 
For all synthesized molecules, only one regioisomer could be detected by ${ }^{1} \mathrm{H}-\mathrm{NMR}$. Only one singlet was observed in the ${ }^{1} \mathrm{H}-\mathrm{NMR}$ spectrum for the triazole ring $(\delta 7.19-8.16 \mathrm{ppm})$, which can be attributed to the proton in the $\mathrm{C}-5$ position of the triazole nucleus. According to earlier literature on copper catalyzed cycloadditions [24,25], it is believed that the obtained products are 1,4-regioisomers.

\subsection{Biology}

For each experiment, inocula controls produced clearly detectable growth after the chosen incubation period, indicating that all isolates were viable and that the conditions used were suitable for fungal growth. The antifungal screening results by MIC measurements are summarized in Table 1 . Most of the synthesized 1,2,3-triazoles showed weak $(\mathbf{4 a}, \mathbf{b}, \mathbf{d}, \mathbf{e}, \mathbf{f})$ or no activity $(\mathbf{4 g}, \mathbf{h}, \mathbf{i}, \mathbf{j})$ against the Candida species used herein. However, for 2-[1-(4-chlorophenyl)-1H-(1,2,3)triazol-4-yl]propan-2-ol (4c) and for the reference drug (fluconazole), it was possible to determine a MIC for Candida growth. As it can be seen, fluconazole showed fungistatic activity in concentrations ranging from 0.5 to $64 \mu \mathrm{g} \cdot \mathrm{mL}^{-1}$. Eight isolates (4987, 4986, 4984, 4970, 4790, 4608, 1059 and 109) had their growth inhibited in a dose-dependent pattern, presenting MIC values ranging from 16 to $32 \mu \mathrm{g} \cdot \mathrm{mL}^{-1}$. In contrast to these findings, nine isolates (4990, 4802, 4388, 4263, 4261, 4124, 3719, 1150 and 934) were resistant to the reference azole showing MIC values above $64 \mu \mathrm{g} \cdot \mathrm{mL}^{-1}$. It can be also observed that the triazole $4 \mathbf{c}$ showed good antifungal activity, presenting MIC values ranging from 64 to $256 \mu \mathrm{g} \cdot \mathrm{mL}^{-1}$ against all the tested strains.

Table 1. Antifungal activity of triazoles $\mathbf{4 a}-\mathbf{j}$ and fluconazole against the studied Candida strains.

\begin{tabular}{ccccccccccccc}
\hline $\begin{array}{c}\text { Tested strain } \\
\mathbf{n}^{\circ} \text { URM }\end{array}$ & $\mathbf{1 0}$ & $\mathbf{4 a}$ & $\mathbf{4 b}$ & $\mathbf{4 c}$ & $\mathbf{4 d}$ & $\mathbf{4 e}$ & $\mathbf{4 f}$ & $\mathbf{4 g}$ & $\mathbf{4 h}$ & $\mathbf{4 i}$ & $\mathbf{4 j}$ & $\mathbf{F l u}^{\mathbf{b}}$ \\
\hline 4990 & 2,048 & 1,024 & 256 & 2,048 & 1,024 & 2,048 & $\mathrm{R}$ & $\mathrm{R}$ & $\mathrm{R}$ & $\mathrm{R}$ & $>64$ \\
4987 & 2,048 & 1,024 & 128 & 2,048 & 1,024 & 2,048 & $\mathrm{R}$ & $\mathrm{R}$ & $\mathrm{R}$ & $\mathrm{R}$ & 16 \\
4986 & 2,048 & 1,024 & 128 & 2,048 & 1,024 & 2,048 & $\mathrm{R}$ & $\mathrm{R}$ & $\mathrm{R}$ & $\mathrm{R}$ & 16 \\
4820 & 2,048 & 1,024 & 256 & 2,048 & 1,024 & 2,048 & $\mathrm{R}$ & $\mathrm{R}$ & $\mathrm{R}$ & $\mathrm{R}$ & 4 \\
4819 & 2,048 & 1,024 & 128 & 1,024 & 1,024 & 2,048 & $\mathrm{R}$ & $\mathrm{R}$ & $\mathrm{R}$ & $\mathrm{R}$ & 4 \\
4817 & 2,048 & 1,024 & 128 & 1,024 & 1,024 & 2,048 & $\mathrm{R}$ & $\mathrm{R}$ & $\mathrm{R}$ & $\mathrm{R}$ & 4 \\
4609 & 2,048 & 1,024 & 128 & 1,024 & 1,024 & 2,048 & $\mathrm{R}$ & $\mathrm{R}$ & $\mathrm{R}$ & $\mathrm{R}$ & 4 \\
4606 & 2,048 & 1,024 & 128 & 1,024 & 1,024 & 2,048 & $\mathrm{R}$ & $\mathrm{R}$ & $\mathrm{R}$ & $\mathrm{R}$ & 2 \\
4388 & 2,048 & 1,024 & 128 & 1,024 & 1,024 & 2,048 & $\mathrm{R}$ & $\mathrm{R}$ & $\mathrm{R}$ & $\mathrm{R}$ & $>64$ \\
4387 & 2,048 & 1,024 & 128 & 1,024 & 1,024 & 2,048 & $\mathrm{R}$ & $\mathrm{R}$ & $\mathrm{R}$ & $\mathrm{R}$ & 2 \\
4386 & 2,048 & 1,024 & 128 & 2,048 & 1,024 & 2,048 & $\mathrm{R}$ & $\mathrm{R}$ & $\mathrm{R}$ & $\mathrm{R}$ & 1 \\
4385 & 2,048 & 1,024 & 128 & 1,024 & 1,024 & 2,048 & $\mathrm{R}$ & $\mathrm{R}$ & $\mathrm{R}$ & $\mathrm{R}$ & 2 \\
4384 & 2,048 & 1,024 & 128 & 1,024 & 1,024 & 2,048 & $\mathrm{R}$ & $\mathrm{R}$ & $\mathrm{R}$ & $\mathrm{R}$ & 2 \\
4260 & 2,048 & 1,024 & 128 & 1,024 & 1,024 & 2,048 & $\mathrm{R}$ & $\mathrm{R}$ & $\mathrm{R}$ & $\mathrm{R}$ & 2 \\
4127 & $\mathrm{R}$ & 1,024 & 128 & 1,024 & 2,048 & 2,048 & $\mathrm{R}$ & $\mathrm{R}$ & $\mathrm{R}$ & $\mathrm{R}$ & 0.5 \\
4126 & 2,048 & 1,024 & 128 & 1,024 & 1,024 & 2,048 & $\mathrm{R}$ & $\mathrm{R}$ & $\mathrm{R}$ & $\mathrm{R}$ & 0.5 \\
4125 & 2,048 & 1,024 & 256 & 1,024 & 1,024 & 2,048 & $\mathrm{R}$ & $\mathrm{R}$ & $\mathrm{R}$ & $\mathrm{R}$ & 0.5 \\
4124 & 2,048 & 1,024 & 256 & 2,048 & 1,024 & 2,048 & $\mathrm{R}$ & $\mathrm{R}$ & $\mathrm{R}$ & $\mathrm{R}$ & $>64$ \\
\hline
\end{tabular}


Table 1. Cont.

\begin{tabular}{|c|c|c|c|c|c|c|c|c|c|c|c|}
\hline \multirow{2}{*}{$\begin{array}{c}\text { Tested strain } \\
\mathbf{n}^{\circ} \text { URM }\end{array}$} & \multicolumn{11}{|c|}{ Compounds (MICs in $\left.\mu \mathrm{g} \cdot \mathrm{mL}^{-1}\right)^{\mathrm{a}}$} \\
\hline & $4 \mathbf{a}$ & $4 b$ & $4 c$ & $4 d$ & $4 e$ & $4 f$ & $4 g$ & $4 h$ & $4 \mathbf{i}$ & $4 \mathbf{j}$ & Flu ${ }^{b}$ \\
\hline 3719 & $\mathrm{R}$ & 1,024 & 256 & 2,048 & 1,024 & 2,048 & $\mathrm{R}$ & $\mathrm{R}$ & $\mathrm{R}$ & $\mathrm{R}$ & 64 \\
\hline 3716 & $\mathrm{R}$ & 1,024 & 256 & 2,048 & 1,024 & 2,048 & $\mathrm{R}$ & $\mathrm{R}$ & $\mathrm{R}$ & $\mathrm{R}$ & 0.5 \\
\hline 4802 & 2,048 & 2,048 & 256 & 2,048 & 1,024 & $\mathrm{R}$ & $\mathrm{R}$ & $\mathrm{R}$ & $\mathrm{R}$ & $\mathrm{R}$ & 64 \\
\hline 4263 & 2,048 & 2,048 & 256 & 2,048 & 1,024 & $\mathrm{R}$ & $\mathrm{R}$ & $\mathrm{R}$ & $\mathrm{R}$ & $\mathrm{R}$ & 64 \\
\hline 1059 & 2,048 & 1,024 & 64 & 512 & 1,024 & 2,048 & $\mathrm{R}$ & $\mathrm{R}$ & $\mathrm{R}$ & $\mathrm{R}$ & 16 \\
\hline 934 & 2,048 & 2,048 & 256 & 2,048 & 1,024 & $\mathrm{R}$ & $\mathrm{R}$ & $\mathrm{R}$ & $\mathrm{R}$ & $\mathrm{R}$ & 64 \\
\hline 109 & 2,048 & 1,024 & 128 & 1,024 & 1,024 & 2,048 & $\mathrm{R}$ & $\mathrm{R}$ & $\mathrm{R}$ & $\mathrm{R}$ & 16 \\
\hline 4984 & $\mathrm{R}$ & 2,048 & 128 & 1,024 & 1,024 & 2,048 & $\mathrm{R}$ & $\mathrm{R}$ & $\mathrm{R}$ & $\mathrm{R}$ & 16 \\
\hline 4970 & $\mathrm{R}$ & 2,048 & 128 & 1,024 & 512 & 2,048 & $\mathrm{R}$ & $\mathrm{R}$ & $\mathrm{R}$ & $\mathrm{R}$ & 16 \\
\hline 4889 & $\mathrm{R}$ & 1,024 & 64 & 1,024 & 1,024 & 2,048 & $\mathrm{R}$ & $\mathrm{R}$ & $\mathrm{R}$ & $\mathrm{R}$ & 2 \\
\hline 4818 & $\mathrm{R}$ & 2,048 & 128 & 1,024 & 1,024 & 2,048 & $\mathrm{R}$ & $\mathrm{R}$ & $\mathrm{R}$ & $\mathrm{R}$ & 4 \\
\hline 4804 & $\mathrm{R}$ & 2,048 & 128 & 1,024 & 1,024 & 2,048 & $\mathrm{R}$ & $\mathrm{R}$ & $\mathrm{R}$ & $\mathrm{R}$ & 8 \\
\hline 4608 & $\mathrm{R}$ & 1,024 & 128 & 1,024 & 1,024 & 2,048 & $\mathrm{R}$ & $\mathrm{R}$ & $\mathrm{R}$ & $\mathrm{R}$ & 16 \\
\hline 4607 & $\mathrm{R}$ & 1,024 & 128 & 1,024 & 1,024 & 2,048 & $\mathrm{R}$ & $\mathrm{R}$ & $\mathrm{R}$ & $\mathrm{R}$ & 4 \\
\hline 4261 & $\mathrm{R}$ & 2,048 & 256 & 1,024 & 1,024 & $\mathrm{R}$ & $\mathrm{R}$ & $\mathrm{R}$ & $\mathrm{R}$ & $\mathrm{R}$ & $>64$ \\
\hline 3627 & 2,048 & 1,024 & 64 & 1,024 & 1,024 & 2,048 & $\mathrm{R}$ & $\mathrm{R}$ & $\mathrm{R}$ & $\mathrm{R}$ & 0.5 \\
\hline 3624 & 2,048 & 1,024 & 64 & 1,024 & 1,024 & 2,048 & $\mathrm{R}$ & $\mathrm{R}$ & $\mathrm{R}$ & $\mathrm{R}$ & 0.5 \\
\hline 3621 & 2,048 & 1,024 & 64 & 1,024 & 1,024 & 2,048 & $\mathrm{R}$ & $\mathrm{R}$ & $\mathrm{R}$ & $\mathrm{R}$ & 0.5 \\
\hline $22019^{c}$ & $\mathrm{R}$ & 2,048 & 256 & 2,048 & 1,024 & 2,048 & $\mathrm{R}$ & $\mathrm{R}$ & $\mathrm{R}$ & $\mathrm{R}$ & 8 \\
\hline 4790 & $\mathrm{R}$ & 2,048 & 128 & 2,048 & 1,024 & 2,048 & $\mathrm{R}$ & $\mathrm{R}$ & $\mathrm{R}$ & $\mathrm{R}$ & 32 \\
\hline 4262 & $\mathrm{R}$ & 1,024 & 128 & 2,048 & 1,024 & 2,048 & $\mathrm{R}$ & $\mathrm{R}$ & $\mathrm{R}$ & $\mathrm{R}$ & 4 \\
\hline 1150 & $\mathrm{R}$ & 2,048 & 128 & 2,048 & 1,024 & 2,048 & $\mathrm{R}$ & $\mathrm{R}$ & $\mathrm{R}$ & $\mathrm{R}$ & $>64$ \\
\hline 933 & $\mathrm{R}$ & 2,048 & 128 & 2,048 & 1,024 & 2,048 & $\mathrm{R}$ & $\mathrm{R}$ & $\mathrm{R}$ & $\mathrm{R}$ & 4 \\
\hline 916 & $\mathrm{R}$ & 2,048 & 128 & 2,048 & 1,024 & 2,048 & $\mathrm{R}$ & $\mathrm{R}$ & $\mathrm{R}$ & $\mathrm{R}$ & 4 \\
\hline
\end{tabular}

${ }^{\mathrm{a}}$ The MIC value was defined as the lowest concentration of the antifungal agent and were read after two days at $37{ }^{\circ} \mathrm{C}$. Inocula sizes contained approximately $2.5 \times 10^{3}$ cells $\cdot \mathrm{mL}^{-1}$. Culture media tested were the RPMI 1640 (Sigma Chemical Co., St. Louis, MO, USA). The final concentration of triazoles was between $4-2,048 \mu \mathrm{g} \cdot \mathrm{mL}^{-1}$ and $0.125-64 \mu \mathrm{g} \cdot \mathrm{mL}^{-1}$ for fluconazole; ${ }^{\mathrm{b}}$ Fluconazole;

${ }^{\mathrm{c}}$ Candida parapsilosis ATCC 22019 was used as reference strain. $\mathrm{R}=$ Resistance.

Observing the drug structure, it can be noticed that $\mathbf{4 c}$ possesses a chlorine atom substituted in the para position of the phenyl ring present at the $N-1$ position of the triazole nucleus. Chlorine-substituted rings were found to be good antifungal tools, as reported by Wang and colleagues [26]. In their study, sixteen $\mathrm{N}$-methyl-substituted phenoxybutan-1-amine chloro-substituted derivatives exhibited strong in vitro antifungal activity, being more active against the tested microorganisms than the used reference drug, voriconazole. Later, Wang et al. [27] decided to synthesize fourteen novel triazolesubstituted compounds containing a phenoxyalkyl group. They also observed that the best antifungal drugs were those with halogen atoms as substituents in the phenyl rings.

The acute preliminary toxicological tests in rats showed that the oral administration of triazole $\mathbf{4 c}$ at the $2,000 \mathrm{mg} \cdot \mathrm{kg}^{-1}$ dose did not produce any signs of toxicity or mortality, indicating that the lethal dose for $50 \%$ of the animal population in this study $\left(\mathrm{LD}_{50}\right)$ is above $2,000 \mathrm{mg} \cdot \mathrm{kg}^{-1}$. According to Lorke [28], substances presenting a $\mathrm{LD}_{50}$ higher than $2,000 \mathrm{mg} \cdot \mathrm{kg}^{-1}$ can be considered low toxicity 
drugs. Since our pharmacological studies have shown that $4 \mathbf{c}$ is active in 64 to $256 \mu \mathrm{g} \cdot \mathrm{mL}^{-1}$ concentrations, those toxicological findings demonstrate that this drug candidate is quite safe for further in vivo studies and can be considered an an antifungal lead for this class of compounds.

\section{Experimental}

\subsection{General}

All commercially available reagents were used without any further purification and the reactions were monitored by TLC analysis (TLC plates $\mathrm{GF}_{254}$ E. Merck). Melting points were determined on a Büchi apparatus and are uncorrected. Column chromatography was performed on Silica Gel 60 (70-230 mesh, Merck Chemicals International). NMR spectra were recorded with a Bruker AC-200 $\mathrm{MHz}$ spectrometer (Billerica, MA, USA) and referenced as follows: ${ }^{1} \mathrm{H}(200 \mathrm{MHz})$, internal $\mathrm{SiMe}_{4}$ at $\delta=0.00 \mathrm{ppm},{ }^{13} \mathrm{C}(50 \mathrm{MHz})$, internal standard at $\delta=77.23 \mathrm{ppm}$. Exact mass measurements of the molecular ions were obtained on a Shimadzu LC/MS-IT-TOF Eletrospray.

\subsection{Synthesis of the Aromatic Azides $\mathbf{2 a}-\mathbf{j}$}

To a solution of the corresponding aniline $\mathbf{1 a}-\mathbf{j}(4.1 \mathrm{mmol})$ dissolved in $\mathrm{CH}_{2} \mathrm{Cl}_{2}(30 \mathrm{~mL})$, was added $6 \mathrm{~N} \mathrm{HCl}(30 \mathrm{~mL})$ at $0{ }^{\circ} \mathrm{C}$. To this biphasic system was added dropwise a saturated aqueous solution of $\mathrm{NaNO}_{2}(10 \mathrm{~mL})$. After stirring for $30 \mathrm{~min}$ at $0{ }^{\circ} \mathrm{C}, \mathrm{NaN}_{3}(0.53 \mathrm{~g}, 8.2 \mathrm{mmol})$ was added at $0{ }^{\circ} \mathrm{C}$. Stirring was maintained for $30 \mathrm{~min}$, and the mixture was allowed to warm to room temperature. The two phases were separated, and the aqueous phase was extracted with $\mathrm{CH}_{2} \mathrm{Cl}_{2}(3 \times 20 \mathrm{~mL})$. The combined organic layers were washed with aqueous solution of $\mathrm{NaHCO}_{3}$, then brine, dried $\left(\mathrm{Na}_{2} \mathrm{SO}_{4}\right)$ and filtered from active charcoal. Evaporation of the solvent in vacuo gave the crude azides $\mathbf{2 a}-\mathbf{j}$ that were used in the next step without further purification.

\subsection{Synthesis of 2-(1-Aryl-1H-1,2,3triazol-4-yl)propan-2-ols $\mathbf{4 a - j}$}

2-Methylbut-3-yn-2-ol (3, 1,1 mmol) and the azido compound $\mathbf{2 a - j}(1 \mathrm{mmol})$ were suspended in a 1:1 mixture of $\mathrm{CH}_{2} \mathrm{Cl}_{2}$ and water $(10 \mathrm{~mL})$. To this solution was added a mixture of $\mathrm{Cu}(\mathrm{OAc})_{2}(36 \mathrm{mg}$, $0.2 \mathrm{mmol}$ ) and sodium ascorbate $(79 \mathrm{mg}, 0.4 \mathrm{mmol})$. The resulting mixture was stirred at room temperature until TLC analysis indicated complete consumption of the azide. The mixture was diluted with $\mathrm{CH}_{2} \mathrm{Cl}_{2}(5 \mathrm{~mL})$ and water $(5 \mathrm{~mL})$. The organic layer was separated, and the water phase was extracted again with $\mathrm{CH}_{2} \mathrm{Cl}_{2}(5 \mathrm{~mL})$. The combined organic layers were dried over $\mathrm{Na}_{2} \mathrm{SO}_{4}$. Removal of the solvent in vacuo gave a residue that was recrystallized from chloroform-hexanes to afford the corresponding triazoles $\mathbf{4 a}-\mathbf{j}$.

2-(1-Phenyl-1H-1,2,3-triazol-4-yl)propan-2-ol (4a): White crystals; yield 86\%; m.p.: 95-96 ${ }^{\circ} \mathrm{C} ; R_{\mathrm{f}}$ 0,60 (ethyl acetate-chloroform 9:1, v/v). ${ }^{1} \mathrm{H}-\mathrm{NMR}\left(\mathrm{CDCl}_{3}\right): \delta 0.97(6 \mathrm{H}, \mathrm{s}) ; 2.92(1 \mathrm{H}, \mathrm{bs}) ; 6.73$ $(3 \mathrm{H}, \mathrm{m}) ; 6.93(1 \mathrm{H}, \mathrm{dd}, J=8 \mathrm{~Hz}, 2 \mathrm{~Hz}) ; 6.97(1 \mathrm{H}, \mathrm{dd}, J=8 \mathrm{~Hz}, 2 \mathrm{~Hz}) ; 7.24(1 \mathrm{H}, \mathrm{s}) .{ }^{13} \mathrm{C}-\mathrm{NMR}\left(\mathrm{CDCl}_{3}\right)$ : $\delta \quad 30.3 ; 68.5 ; 117.7 ; 120.4 ; 128.5 ; 129.6 ; 136.9 ; 156.4$. ESI-HRMS $\mathrm{m} / z$ : 226.0911 (calcd. for $\mathrm{C}_{11} \mathrm{H}_{13} \mathrm{~N}_{3} \mathrm{ONa}[\mathrm{M}+\mathrm{Na}]^{+}:$226.0956). 
2-[1-(4-Tolyl)-1H-1,2,3-triazol-4-yl]propan-2-ol (4b): White crystals; yield 78\%; m.p.: 120-121 ${ }^{\circ} \mathrm{C}$; $R_{\mathrm{f}} 0,65$ (ethyl acetate-chloroform 9:1, v/v). ${ }^{1} \mathrm{H}-\mathrm{NMR}\left(\mathrm{CDCl}_{3}\right): \delta 0.99(6 \mathrm{H}, \mathrm{s}) ; 1.68(3 \mathrm{H}, \mathrm{s}) ; 3.13(1 \mathrm{H}$, bs); $6.54(2 \mathrm{H}, \mathrm{bd}, J=8.0 \mathrm{~Hz}) ; 6.85(2 \mathrm{H}, \mathrm{bd}, J=8.0 \mathrm{~Hz}) ; 7.24(1 \mathrm{H}, \mathrm{s}) .{ }^{13} \mathrm{C}-\mathrm{NMR}\left(\mathrm{CDCl}_{3}\right): \delta 20.9 ; 30.4$; $68.4 ; 117.7 ; 120.2 ; 130.0 ; 134.6 ; 138.5 ; 156.2$. ESI-HRMS $m / z: 240.1049$ (calcd. for $\mathrm{C}_{12} \mathrm{H}_{15} \mathrm{~N}_{3} \mathrm{ONa}$ $\left.[\mathrm{M}+\mathrm{Na}]^{+}: 240.1113\right)$.

2-[1-(4-Chlorophenyl)-1H-1,2,3-triazol-4-yl]propan-2-ol (4c): White crystals; yield 67\%; m.p.: 93-94 ${ }^{\circ} \mathrm{C} ; R_{\mathrm{f}} 0,67$ (ethyl acetate-chloroform 9:1, v/v). ${ }^{1} \mathrm{H}-\mathrm{NMR}\left(\mathrm{CDCl}_{3}\right): \delta 0.98(6 \mathrm{H}, \mathrm{s}) ; 2.90(1 \mathrm{H}, \mathrm{bs})$; $6.75(2 \mathrm{H}, \mathrm{dd}, J=8.0 \mathrm{~Hz}, 2 \mathrm{~Hz}) ; 6.93(2 \mathrm{H}, \mathrm{dd}, J=8.0 \mathrm{~Hz}, 2.0 \mathrm{~Hz}) ; 7.24(1 \mathrm{H}, \mathrm{s}) .{ }^{13} \mathrm{C}-\mathrm{NMR}\left(\mathrm{CDCl}_{3}\right): \delta$ $30.3 ; 68.5 ; 107.1 ; 117.6 ; 121.5 ; 129.7 ; 134.2 ; 135.4 ; 156.6$. ESI-HRMS $\mathrm{m} / \mathrm{z}: 260.0518$ (calcd. for $\left.\mathrm{C}_{11} \mathrm{H}_{12} \mathrm{ClN}_{3} \mathrm{ONa}[\mathrm{M}+\mathrm{Na}]^{+}: 260,0567\right)$.

2-[1-(4-Bromophenyl)-1H-1,2,3-triazol-4-yl]propan-2-ol (4d): White crystals; yield 96\%; m.p.: 95-96 ${ }^{\circ} \mathrm{C} ; R_{\mathrm{f}} 0,68$ (ethyl acetate-chloroform 9:1, v/v). ${ }^{1} \mathrm{H}-\mathrm{NMR}\left(\mathrm{CDCl}_{3}\right): \delta 1.70(6 \mathrm{H}, \mathrm{s}) ; 3.68(1 \mathrm{H}, \mathrm{bs})$; $7.59(4 \mathrm{H}, \mathrm{m}) ; 7.97(1 \mathrm{H}, \mathrm{s}) .{ }^{13} \mathrm{C}-\mathrm{NMR}\left(\mathrm{CDCl}_{3}\right): \delta 30.3 ; 68.4 ; 107.0 ; 121.7 ; 122.1 ; 132.7 ; 135.8 ; 156.7$. ESI-HRMS m/z: 305.9989 (calcd. for $\mathrm{C}_{11} \mathrm{H}_{12} \mathrm{BrN}_{3} \mathrm{ONa}[\mathrm{M}+\mathrm{Na}]^{+}: 304.0061$ ).

2-[1-(4-Methoxyphenyl)-1H-1,2,3-triazol-4-yl]propan-2-ol (4e): Red crystals; yield 69\%; m.p.: 106-107 ${ }^{\circ} \mathrm{C} ; R_{\mathrm{f}} 0,65$ (ethyl acetate-chloroform 9:1, v/v). ${ }^{1} \mathrm{H}-\mathrm{NMR}\left(\mathrm{CDCl}_{3}\right): \delta 1.67(6 \mathrm{H}, \mathrm{s}) ; 3.00(1 \mathrm{H}$, bs); $3.82(3 \mathrm{H}, \mathrm{s}) ; 6.96(2 \mathrm{H}, \mathrm{dd}, J=6.0 \mathrm{~Hz}, 4.0 \mathrm{~Hz}) ; 7.57(2 \mathrm{H}, \mathrm{dd} J=6.0 \mathrm{~Hz}, 4.0 \mathrm{~Hz}) ; 7.81(1 \mathrm{H}, \mathrm{s})$. ${ }^{13} \mathrm{C}-\mathrm{NMR}\left(\mathrm{CDCl}_{3}\right): \delta 30.4 ; 55.5 ; 68.5 ; 114.6 ; 122.1 ; 130.5 ; 159.6$. ESI-HRMS m/z: 238.0757 (calcd. for $\left.\mathrm{C}_{12} \mathrm{H}_{13} \mathrm{ClNO}_{2}[\mathrm{M}+\mathrm{H}]^{+}: 238.0635\right)$.

2-[1-(3-Nitrophenyl)-1H-1,2,3-triazol-4-yl]propan-2-ol (4f): White crystals; yield 75\%; m.p.: 98-100 ${ }^{\circ} \mathrm{C} ; R_{\mathrm{f}} 0,60$ (ethyl acetate-chloroform 9:1, v/v). ${ }^{1} \mathrm{H}-\mathrm{NMR}\left(\mathrm{CDCl}_{3}\right): \delta 1.63(6 \mathrm{H}, \mathrm{s}) ; 3.96(1 \mathrm{H}$, bs); $7.65(1 \mathrm{H}, \mathrm{bd}, J=8.0 \mathrm{~Hz}) ; 8.12(2 \mathrm{H}, \mathrm{m}) ; 8.16(1 \mathrm{H}, \mathrm{s}) ; 7.65(1 \mathrm{H}, \mathrm{d}, J=2.1 \mathrm{~Hz}) .{ }^{13} \mathrm{C}-\mathrm{NMR}\left(\mathrm{CDCl}_{3}\right)$ : $\delta 30.2 ; 68.4 ; 114.9 ; 122.9 ; 125.8 ; 130.8 ; 137.5 ; 148.6 ; 157.1$. ESI-HRMS $m / z: 271.0764$ (calcd. for $\left.\mathrm{C}_{11} \mathrm{H}_{12} \mathrm{~N}_{4} \mathrm{O}_{3} \mathrm{Na}[\mathrm{M}+\mathrm{Na}]^{+}: 271.0807\right)$.

2-[1-(4-Nitrophenyl)-1H-1,2,3-triazol-4-yl]propan-2-ol (4g): Yellow crystals; yield 58\%; m.p.: 123-124 ${ }^{\circ} \mathrm{C} ; R_{\mathrm{f}} 0,63$ (ethyl acetate-chloroform 9:1, v/v). ${ }^{1} \mathrm{H}-\mathrm{NMR}\left(\mathrm{CDCl}_{3}\right): \delta 1.69(6 \mathrm{H}, \mathrm{s}) ; 2.82(1 \mathrm{H}$, bs); $7.95(2 \mathrm{H}, \mathrm{dd}, J=6.0 \mathrm{~Hz}, 4.0 \mathrm{~Hz}) ; 8.03(1 \mathrm{H}, \mathrm{s}) ; 8.37(2 \mathrm{H}, \mathrm{dd}, J=6.0 \mathrm{~Hz}, 2.0 \mathrm{~Hz}) .{ }^{13} \mathrm{C}-\mathrm{NMR}$ $\left(\mathrm{CDCl}_{3}\right): \delta 30.4 ; 68.7 ; 107.1 ; 120.3 ; 125.5 ; 141.2 ; 147.0 ; 157.2$. ESI-HRMS $m / z: 265.1469$ (calcd. for $\mathrm{C}_{11} \mathrm{H}_{13} \mathrm{~N}_{4} \mathrm{O}_{4}\left[\mathrm{M}+\mathrm{H}_{2} \mathrm{O}-\mathrm{H}\right]^{+}:$265.2453).

2-[1-(3,4-Dimethylphenyl)-1H-1,2,3-triazol-4-yl]propan-2-ol (4h): yellow crystals; yield 31\%; m.p.: 128-129 ${ }^{\circ} \mathrm{C} ; R_{\mathrm{f}} 0,80$ (ethyl acetate:chloroform 9:1, v/v). ${ }^{1} \mathrm{H}-\mathrm{NMR}\left(\mathrm{CDCl}_{3}\right): \delta 1.00(6 \mathrm{H}, \mathrm{s}) ; 1.60$ $(6 \mathrm{H}, \mathrm{s}) ; 2.69(1 \mathrm{H}, \mathrm{bs}) ; 6.53(1 \mathrm{H}, \mathrm{m}) ; 6.72(2 \mathrm{H}, \mathrm{dd}, J=8.1 \mathrm{~Hz}, 2.0 \mathrm{~Hz}) ; 7.19(1 \mathrm{H}, \mathrm{s}) .{ }^{13} \mathrm{C}-\mathrm{NMR}$ $\left(\mathrm{CDCl}_{3}\right): \delta 19.3 ; 19.8 ; 30.4 ; 68.5 ; 107.1 ; 117.7 ; 121.5 ; 130.4 ; 134.9 ; 137.2 ; 138.1 ; 156.0$. ESI-HRMS $m / z: 254.1229$ (Calcd for $\mathrm{C}_{13} \mathrm{H}_{17} \mathrm{~N}_{3} \mathrm{ONa}[\mathrm{M}+\mathrm{Na}]^{+}: 254.1269$ ).

2-[1-( $\alpha$-Naphthyl)-1H-1,2,3-triazol-4-yl]propan-2-ol (4i): Red crystals; yield 62\%; m.p.: 152-153 ${ }^{\circ} \mathrm{C}$; $R_{\mathrm{f}} 0,65$ (ethyl acetate:chloroform 9:1, v/v). ${ }^{1} \mathrm{H}-\mathrm{NMR}\left(\mathrm{CDCl}_{3}\right): \delta 1.75(6 \mathrm{H}, \mathrm{s}) ; 3.00(1 \mathrm{H}, \mathrm{bs}) ; 7.54$ $(4 \mathrm{H}, \mathrm{m}) ; 7.82(1 \mathrm{H}, \mathrm{s}) ; 7.95(3 \mathrm{H}, \mathrm{m}) .{ }^{13} \mathrm{C}-\mathrm{NMR}\left(\mathrm{CDCl}_{3}\right): \delta 30.5 ; 68.6 ; 122.3 ; 123.5 ; 127.0 ; 128.2$; 
128.5; 130.3; 133.7; 134.0; 155.5. ESI-HRMS $m / z: 276.1070$ (calcd. for $\mathrm{C}_{15} \mathrm{H}_{15} \mathrm{~N}_{3} \mathrm{ONa}[\mathrm{M}+\mathrm{Na}]^{+}$: 276.1113).

2-[1-( $\beta$-Naphthyl)-1H-1,2,3-triazol-4-yl]propan-2-ol (4j): Yellow crystals; yield 60\%; m.p.: 143-144 ${ }^{\circ} \mathrm{C} ; R_{\mathrm{f}} 0,68$ (ethyl acetate:chloroform 9:1, v/v). ${ }^{1} \mathrm{H}-\mathrm{NMR}\left(\mathrm{CDCl}_{3}\right): \delta 1.72(6 \mathrm{H}, \mathrm{s}) ; 2.97(1 \mathrm{H}$, bs); $7.53(2 \mathrm{H}, \mathrm{d}, J=8.1 \mathrm{~Hz}) ; 7.89(4 \mathrm{H}, \mathrm{m}) ; 8.03(1 \mathrm{H}, \mathrm{s}) ; 8.12(1 \mathrm{H}, \mathrm{d}, J=2.1 \mathrm{~Hz}) .{ }^{13} \mathrm{C}-\mathrm{NMR}\left(\mathrm{CDCl}_{3}\right)$ : $\delta 30.4 ; 68.6 ; 118.3 ; 118.9 ; 126.9 ; 127.3 ; 127.8 ; 128.2 ; 129.9 ; 132.7 ; 133.1 ; 134.4 ; 155.6$. ESI-HRMS $m / z: 276.1071$ (calcd. for $\mathrm{C}_{15} \mathrm{H}_{15} \mathrm{~N}_{3} \mathrm{ONa}[\mathrm{M}+\mathrm{Na}]^{+}: 276.1113$ ).

\subsection{Strains and Growth Cultures}

Twenty strains of Candida albicans, five of Candida krusei, eleven of Candida parapsilosis and five of Candida tropicalis were supplied by the URM Culture Collection of the Department of Mycology, Biological Sciences Centre of the Federal University of Pernambuco, Recife, Brazil. Strains have been stocked in mineral oil at $18{ }^{\circ} \mathrm{C}$ [28]. Viability tests and subsequent taxonomic confirmation of their morphological, biochemical and physiological characteristics were carried out [29]. Species, accession numbers, stock time and isolation substratum are summarized in Table 2.

Table 2. Samples of Candida species preserved in the Mycotheca Culture CollectionUniversity of Recife Mycology (URM).

\begin{tabular}{lccc}
\hline Species & Accession $\mathbf{n}^{\mathbf{0}}$ (URM) & Storage (years) & Substratum \\
\hline C. albicans & 4990 & 01 & Vaginal secretion \\
C. albicans & 4987 & 01 & Vaginal secretion \\
C. albicans & 4986 & 01 & Vaginal secretion \\
C. albicans & 4820 & 02 & Ungual scrap \\
C. albicans & 4819 & 02 & Ungual scrap \\
C. albicans & 4817 & 02 & Ungual scrap \\
C. albicans & 4609 & 03 & Blood \\
C. albicans & 4606 & 03 & Blood \\
C. albicans & 4388 & 05 & Oropharyngeal secretion \\
C. albicans & 4387 & 05 & Oropharyngeal secretion \\
C. albicans & 4386 & 05 & Oropharyngeal secretion \\
C. albicans & 4385 & 05 & Oropharyngeal secretion \\
C. albicans & 4384 & 05 & Oropharyngeal secretion \\
C. albicans & 4260 & 05 & Oropharyngeal secretion \\
C. albicans & 4127 & 07 & Inguinal area \\
C. albicans & 4126 & 07 & Urine \\
C. albicans & 4125 & 07 & Spittle \\
C. albicans & 4124 & 07 & Oropharyngeal secretion \\
C. albicans & 3719 & 10 & Tooth scrap \\
C. albicans & 3716 & 10 & Tooth scrap \\
C. krusei & 4802 & 02 & $*$ \\
C. krusei & 4263 & 05 & Oropharyngeal secretion \\
C. krusei & 1059 & 48 & Appendix biopsy \\
C. krusei & 934 & 49 & \\
\hline
\end{tabular}


Table 2. Cont.

\begin{tabular}{lccc}
\hline Species & Accession $\mathbf{n}^{\mathbf{0}}$ (URM) & Storage (years) & Substratum \\
\hline C. krusei & 109 & 52 & $*$ \\
C. parapsilosis & 4984 & 01 & Vaginal secretion \\
C. parapsilosis & 4970 & 01 & Vaginal secretion \\
C. parapsilosis & 4889 & 02 & Blood \\
C. parapsilosis & 4818 & 02 & Ungual scrap \\
C. parapsilosis & 4804 & 02 & IFM \\
C. parapsilosis & 4608 & 03 & Blood \\
C. parapsilosis & 4607 & 03 & Blood \\
C. parapsilosis & 4261 & 05 & Oropharyngeal secretion \\
C. parapsilosis & 3627 & 12 & Spittle \\
C. parapsilosis & 3624 & 12 & Spittle \\
C. parapsilosis & 3621 & 12 & Spittle \\
C. parapsilosis & ATCC22019 & - & - \\
C. tropicalis & 4790 & 02 & Cassava powdery \\
C. tropicalis & 4262 & 06 & Oropharyngeal secretion \\
C. tropicalis & 1150 & 46 & Tongue \\
C. tropicalis & 933 & 49 & Vaginal secretion \\
C. tropicalis & 916 & 49 & Feces \\
\hline
\end{tabular}

\subsection{In vitro Antifungal Susceptibility}

Reference microdilution trays, containing serial drug dilutions were prepared by following the CLSI M27-A3 guidelines [30]. The triazoles were dissolved in dimethylsulfoxide (DMSO) and then these stock solutions were stored at $-80{ }^{\circ} \mathrm{C}$. The concentrations tested ranged from 2 to $2,048 \mu \mathrm{g} \cdot \mathrm{mL}^{-1}$. Fluconazole was used as reference drug at concentrations from 0.125 to $64 \mu \mathrm{g} \cdot \mathrm{mL}^{-1}$. In order to obtain a fungal inoculum containing $1-5 \times 10^{6} \mathrm{CFU} \cdot \mathrm{mL}^{-1}$, each strain was cultured on a tube containing $20 \mathrm{~mL}$ of Sabouraud Dextrose Agar (SDA) plus yeast extract at $35^{\circ} \mathrm{C}$ for two days. After this time, yeast suspensions were prepared in sterile physiological solution $(0.85 \%)$ and maintained at $28 \pm 2{ }^{\circ} \mathrm{C}$ and then were adjusted to $90 \%$ transmittance at $530 \mathrm{~nm}$. Two serial dilutions from 1:100 and 1:20 sequentially were made to obtain a final inoculum containing $1.0 \times 10^{3}$ and $5 \times 10^{3} \mathrm{CFU} \cdot \mathrm{mL}^{-1}$. The microdilution wells containing $100 \mu \mathrm{L}$ of the twofold serial dilutions of the test and reference drugs in standard RPMI 1640 medium (Sigma Chemical Co., St. Louis, MO, USA), buffered to pH 7.0 with $0.165 \mathrm{~mol} \cdot \mathrm{L}^{-1}$ of morpholinopropanesulphonic acid (MOPS, Sigma), were inoculated with $100 \mu \mathrm{L}$ of inoculum. After inoculation, the microplates were incubated at $35{ }^{\circ} \mathrm{C}$ in a non $\mathrm{CO}_{2}$ incubator and were read visually $48 \mathrm{~h}$ after the incubation. MICs corresponded to the lowest drug dilution that showed growth inhibition compared to untreated yeasts. C. parapsilosis ATCC 22019 was used as reference strain. All tests were performed in triplicate.

\subsection{Animals and Preliminary Toxicological Tests}

Adult male Wistar rats (Rattus norvegicus), aged 2-3 months, weighing 220-260 g, were obtained from the Pound of the Department of Physiology and Pharmacology at the Federal University of 
Pernambuco. They were kept under standard environmental conditions $\left(23 \pm 2{ }^{\circ} \mathrm{C} ; 12: 12 \mathrm{~h}\right.$ dark/light cycle) and water and animal food (Labina ${ }^{\circledR}$, Purina, Brazil) were made available ad libitum. The animals were randomly divided into two groups ( $n=3-4$ /group) and deprived of feed for $12 \mathrm{~h}$ with access to water ad libitum. Further, group 1 received vehicle (solution of 2.5\% tween 80) and group 2 received 2-[1-(4-chlorophenyl)-1H-1,2,3-triazol-4-yl]propan-2-ol (4c) in a single oral dose of $2,000 \mathrm{mg} \cdot \mathrm{kg}^{-1}$. The observations were performed at 30,60, 120, 180 and $240 \mathrm{~min}$ after the oral treatments and then, daily for 14 days. Behavioral changes, weight, consumption of food and water, clinical signs of toxicity, and mortality were recorded daily [31]. The experimental protocol was approved by the Federal University of Pernambuco's Ethics Committee for Animal Experimentation (Process $n^{\circ} 23076.003830$ ). Studies of acute toxicity were performed according to "Up and down" method with slight modifications, as described by OECD 425 [32].

\section{Conclusions}

In conclusion, a series of analogs of 1,2,3-triazoles with ten distinct substituents at the $N-1$ of the triazole ring were synthesized and assessed for their antifungal activity. All compounds were tested against 42 pathogenic strains of four different Candida species. Modification of substituents has a great impact on the minimal inhibitory concentration values, since we could obtain triazole derivatives showing no antimycotic activity, with moderate antifungal activity and one compound with promising activity. The antifungal tests data show that the chloro-substituted triazole derivative exhibited, in particular, good fungal growth inhibition, showing that further modifications in the 2-(1-aryl-1H-1,2,3triazol-4-yl) series can be done in order to obtain more potent prototypes.

\section{Conflict of Interest}

The authors declare no conflict of interest.

\section{Acknowledgments}

The authors gratefully acknowledge CAPES, CNPq, PPP/FACEPE and PRONEX/FACEPE for fellowships and financial support.

\section{References and Notes}

1. Lopez-Martinez, R. Candidosis, a new challenge. Clin. Dermatol. 2010, 6, 178-184.

2. Chen, A.; Sobel, J.D. Emerging azole antifungals. Expert Opin. Emerg. Drugs 2005, 10, 21-33.

3. Rybak, M.J.; Akins, R.L. Emergence of methicillin-resistant Staphylococcus aureus with intermediate lycopeptide resistance: clinical significance and treatment options. Drugs 2001, 6, $1-7$.

4. Sheehan, D.J.; Hitchcock, C.A.; Sibley, C.M. Current and emerging azole antifungal agents. Clin. Microbiol. Rev. 1999, 12, 40-79.

5. Wei, Q.L.; Zhang, S.S.; Gao, J.; Li, W.H.; Xu, L.Z.; Yu, Z.G. Synthesis and QSAR studies of novel triazole compounds containing thioamide as potential antifungal agents. Bioorg. Med. Chem. 2006, 14, 7146-7153. 
6. Warn, P.A.; Sharp, A.; Parmar, A.; Majithiya, J.; Denning, D.W.; Hope, W.W. Pharmacokinetics and pharmacodynamics of a novel triazole, isavuconazole: Mathematical modeling, importance of tissue concentrations, and impact of immune status on antifungal effect. Antimicrob. Agents Chemother. 2009, 53, 3453-3461.

7. Zou, Y.; Zhao, Q.; Liao, J.; Hua, H.; Yu, S.; Chai, X.; Xu, M.; Wua, Q. New triazole derivatives as antifungal agents: Synthesis via click reaction, in vitro evaluation and molecular docking studies. Bioorg. Med. Chem. Lett. 2012, 22, 2959-2962.

8. Ulusoy, N.; Gursoy, A.; Otuk, G. Synthesis and antimicrobial activity of some 1,2,4-triazole-3mercaptoacetic acid derivatives. Farmaco 2001, 56, 947-952.

9. Demirbas, N.; Demirbas, A.; Karaoglu, S.A. Synthesis and biological activities of new 1,2,4-triazol-3-one derivatives. Bioorg. Khim. 2005, 31, 430-440.

10. Bagihalli, G.B.; Avaji, P.G.; Patil, S.A.; Badami, P.S. Synthesis, spectral characterization, in vitro antibacterial, antifungal and cytotoxic activities of $\mathrm{Co}(\mathrm{II}), \mathrm{Ni}(\mathrm{II})$ and $\mathrm{Cu}(\mathrm{II})$ complexes with 1,2,4-triazole Schiff bases. Eur. J. Med. Chem. 2008, 43, 2639-2649.

11. Karthikeyan, M.S.; Prasad, D.J.; Poojary, B.; Bhat, K.S.; Holla, B.S.; Kumari, N.S. Synthesis and biological activity of Schiff and Mannich bases bearing 2,4-dichloro-5-fluorophenyl moiety. Bioorg. Med. Chem. 2006, 14, 7482-7489.

12. Dabak, K.; Sezer, O.; Akar, A.; Anac, O. Synthesis and investigation of tuberculosis inhibition activities of some 1,2,3-triazole derivatives. Eur. J. Med. Chem. 2003, 38, 215-218.

13. Shanmugavelan, P.; Nagarajan, S.; Sathishkumar, M.; Ponnuswamy, A.; Yogeeswari, P.; Sriram, D. Efficient synthesis and in vitro antitubercular activity of 1,2,3-triazolesas inhibitors of Mycobacterium tuberculosis. Bioorg. Med. Chem. Lett. 2011, 21, 7273-7276.

14. Joshi, S.; Khosla, N.; Tiwari, P. In vitro study of some medicinally important Mannich bases derived from antitubercular agent. Bioorg. Med. Chem. 2004, 12, 571-576.

15. Kai, H.; Hinou, H.; Nishimura, S.-I. Aglycone-focused randomization of 2-difluoromethylphenyltype sialoside suicide substrates for neuraminidases. Bioorg. Med. Chem. 2012, 20, 2739-2746.

16. Holla, B.S.; Veerendra, B.; Shivananda, M.K.; Poojary, B. Synthesis, characterization and anticancer activity studies on some Mannich bases derived from 1,2,4-triazoles. Eur. J. Med. Chem. 2003, 38, 759-767.

17. Tiew, K.-C.; Dou, D.; Teramoto, T.; Lai, H.; Alliston, K.R.; Lushington, G.H.; Padmanabhan, R.; Groutas, W.C. Inhibition of Dengue virus and West Nile virus proteases by click chemistryderived benz $[d]$ isothiazol-3(2H)-one derivatives. Bioorg. Med. Chem. 2012, 20, 1213-1221.

18. Turan-Zitouni, G.; Kaplancikli, Z.A.; Erol, K.; Kilic, F.S. Synthesis and analgesic activity of some triazoles and triazolo-thiadiazines. Farmaco 1999, 54, 218-223.

19. He, J.; Feng, L.; Li, J.; Tao, R.; Wang, F.; Liao, X.; Sun, Q.; Long, Q.; Ren, Y.; Wan, J.; He, H. Design, synthesis and biological evaluation of novel 2-methylpyrimidine-4-ylamine derivatives as inhibitors of Escherichia coli pyruvate dehydrogenase complex E1. Bioorg. Med. Chem 2012, 20, $1665-1670$.

20. Oh, K.; Yamada, K.; Asami, T.; Yoshizawa, Y. Synthesis of novel brassinosteroid biosynthesis inhibitors based on the ketoconazole scaffold. Bioorg. Med. Chem. Lett. 2012, 22, 1625-1628. 
21. Malolanarasimhan, K.; Lai, C.C.; Kelley, J.A.; Iaccarino, L.; Reynolds, D.; Young, H.A.; Marquez, V.E. Synthesis and biological study of a flavone acetic acid analogue containing an azido reporting group designed as a multifunctional binding site probe. Bioorg. Med. Chem. 2005, 13, 2717-2722.

22. Lee, B.Y.; Park, S.R.; Jeon, H.B.; Kim, K.S. A new solvent system for efficient synthesis of 1,2,3-triazoles. Tetrahedron Lett. 2006, 47, 5105-5109.

23. dos Anjos, J.V.; Sinou, D.; de Melo, S.J.; Srivastava, R.M. Synthesis of glycosyl-triazole linked 1,2,4-oxadiazoles. Carbohydr. Res. 2007, 342, 2440-2449.

24. Himo, F.; Lovell, T.; Hilgraf, H.; Rostovtsev, V.V.; Noodleman, L.; Sharpless, K.B.; Fokin, V.V. Copper(I)-catalyzed synthesis of azoles. DFT study predicts unprecedented reactivity and intermediates. J. Am. Chem. Soc. 2005, 127, 210-216.

25. dos Anjos, J.V.; Neves Filho, R.A.W.; Nascimento, S.C.; Srivastava, R.M.; Melo, S.J.; Sinou, D. Synthesis and cytotoxic profile of glycosyl triazole linked to 1,2,4-oxadiazole moiety at C-5 through a straight-chain carbon and oxygen atoms. Eur. J. Med. Chem. 2009, 44, 3571-3576.

26. Wang, W.; Sheng, C.; Che, X.; Ji, H.; Cao, Y.; Miao, Z.; Yao, J.; Zhang, W. Discovery of highly potent novel antifungal azoles by structure-based rational design. Bioorg. Med. Chem. Lett. 2009, 19, 5965-5969.

27. Wang, W.; Wang, S.; Liu, Y.; Dong, G.; Cao, Y.; Miao, Z.; Yao, J.; Zhang, W.; Sheng, C. Novel conformationally restricted triazole derivatives with potent antifungal activity. Eur. J. Med. Chem. 2010, 45, 6020-6026.

28. Lorke, D. A new approach to practical acute toxicity testing. Arch. Toxicol. 1983, 54, $275-287$.

29. Sherf, A.F. A method for maintaining Phytomonas sepedomica for long periods without transfer. Phytopathology 1943, 31, 30-32.

30. de Hoog, G.S.; Guarro, J.; Gene, J.; Figueras, M.J. Atlas of Clinical Fungi, 2nd ed.; Centraalbureau voor Schimmelcultures: Utrecht, The Netherlands and Universitat Rovira i Virgili: Reus, Spain, 2000; p. 1124.

30. Clinical Laboratory Standards Institute (CLSI). Reference Method for Broth Dilution Testing of Yeasts; Approved standard-third edition M27-A3; CLSI: Wayne, PA, USA, 2008.

31. Malone, M.H. Pharmacological Approaches to Natural Products Screening and Evaluation. In New Natural Products and Plant Drugs with Pharmacological, Biological or Therapeutical Activity; Wagner, H., Wolf, P., Eds; Springer-Verlag: Berlin, Germany, 1977; pp. 23-53.

32. Organisation for Economic Co-operation and Development (OECD). OECD Guideline for Testing of Chemicals: Acute Oral Toxicity-Up-and-Down Procedure; $\mathrm{n}^{\circ}$ 425; OECD Publishing: Paris, France, 2001.

Sample Availability: Samples of the compounds $\mathbf{4 a - j}$ are available from the authors.

(C) 2012 by the authors; licensee MDPI, Basel, Switzerland. This article is an open access article distributed under the terms and conditions of the Creative Commons Attribution license (http://creativecommons.org/licenses/by/3.0/). 